\title{
Developing and Validating a Virtual Reality Tool for Evaluating Cognitive and Physical Performance during a Simulated Loaded March
}

\section{Itay Ketko}

Heller Institute of Medical Research

Shani Kimel Naor

Sheba Medical Center at Tel Hashomer

Ran Yanovich

Heller Institute of Medical Research

Amihai Gottlieb

Sheba Medical Center at Tel Hashomer

Yotam Bahat

Sheba Medical Center at Tel Hashomer

Oran Ben-Gal

Sheba Medical Center at Tel Hashomer

Yuval Heled

Heller Institute of Medical Research

Meir Plotnik ( $\square$ Meir.PlotnikPeleg@sheba.health.gov.il)

Sheba Medical Center at Tel Hashomer https://orcid.org/0000-0003-2637-3457

\section{Research}

Keywords: physical effort, cognitive load, virtual reality, rescue forces, military, load carriage

Posted Date: April 21st, 2020

DOI: https://doi.org/10.21203/rs.3.rs-22782/v1

License: @ (i) This work is licensed under a Creative Commons Attribution 4.0 International License. Read Full License 


\section{Abstract \\ Background}

Athletes, soldiers, and rescue personnel must often perform intense, prolonged, and physically demanding activities while maintaining cognitive focus. As cognitive and physical functions are believed to share central nervous system resources, their simultaneous activation can cause reciprocal disruptions in the performance of both.

\section{Methods}

In the current study, we aimed to develop and validate a virtual reality-based experimental protocol enabling rigorous exploration of the effects of prolonged high-load physical and cognitive efforts, by comparing novel cognitive tasks presented in the context of a simulated loaded march to a battery of established neurocognitive tests. We then used this protocol in a pre-post pilot study exploring the effects of high-load physical and cognitive activity on physical and cognitive performance. Twelve participants underwent a simulated 10-km loaded march on a treadmill in our virtual reality environment, with or without integrated cognitive tasks (VR-COG). At each of three experimental visits, participants underwent pre-activity and post-activity evaluations, including the Color Trail Test, the Synthetic Work Environment (SYNWIN) battery for multitasking evaluation, and physical tests (i.e., 'time to exhaustion').

\section{Results}

In general, strong or moderate correlations $(r \geq 0.58 p \leq 0.048)$ were found between VR-COG scores and scores on the validated cognitive tests. Together, VRCOG and CTT measures were able to successfully predict the effects of the combined physical and cognitive load on multitasking performance, as assessed by SYNWIN score.

\section{Conclusions}

As virtual environments are ideal for studying high performance professional activity in realistic but controlled settings, the novel protocol is optimal for measuring the effects of prolonged, high-load physical and cognitive activity, and can therefore contribute to our knowledge on physical-cognitive interactions.

\section{Background}

Athletes, soldiers, and rescue personnel must often perform intense, prolonged, and physically demanding activities while maintaining cognitive focus. Under these conditions, they are required to make rapid decisions and process information from multiple sources using different sensory modalities (1, 2). These efforts can cause significant stress, leading to physical and cognitive exhaustion and, in turn, to poor performance. For example, individuals exposed to stressful conditions can experience "perceptual narrowing", during which inattention to perceptual cues leads them to ignore alternatives or make decisions based on incomplete information (3).

It is commonly hypothesized that cognitive and physical functions share central nervous system resources, such that their simultaneous activation can cause reciprocal disruptions in the performance of both $(4,5,6)$. These interactions between physical and cognitive functions are particularly pertinent in the context of military activity, due to critical ramifications for the success of operational missions and for soldier performance and health.

In most cases, pre-post studies examine the effects of exercise on physical $(7,8,9,10)$ or cognitive $(1,11)$ performance separately. Some protocols involve short acute exercise sessions (9) while others examine the effects of prolonged exercise, where fatigue comes into play $(7,8,10,11,12)$. In one such study, Eddy et al. evaluated the effects of load carriage and physical fatigue on cognitive performance. Participants walked for two hours with or without a load while performing two cognitive tasks (auditory go/no-go and visual target detection). With load, performance on both cognitive tasks declined over time (12), showing that physical load can be detrimental to cognitive performance.

Armstrong et al. recently developed a test battery to measure cognitive function in soldiers carrying various loads during long marches (13). Their preliminary data indicate that during loaded marching, cognitive demands reduce the accuracy of soldier responses.

Apart from these findings, there are several voids in the existing literature on the effects of physically and cognitively strenuous activities on functioning in both domains, and on the interactions between them. Most studies have evaluated physical effects without systematically introducing cognitive demands (with the exception of (11)). When included, cognitive functions have generally been assessed using neurocognitive paper-and-pencil tests, rather than mission-oriented challenges tapping a range of relevant competencies, such as executive functions. The ecological validity of these examinations is clearly compromised, prompting further investigation of load carriage effects on cognitive and physical performance in more realistic environments, during exposure to various stressors.

A technology-based solution well-suited to this line of research is virtual reality (VR), which has increasingly been used over the past two decades to study human function (for review (14)) and for clinical purposes $(15,16)$. Large-scale VR laboratories, in particular, can potentially resolve the aforementioned shortcomings, as they enable controlled, systematic, and repeated introduction of cognitive tasks during different degrees of physical exertion (walking on a treadmill incorporated in the VR system). VR technology can also include adaptation of classic pencil-and-paper tests inspecting specific cognitive competencies (e.g., (17)). Essentially, immersion in a realistically designed VR environment (VRE) makes it possible to study behavior under ecologically valid conditions, with laboratory grade control. 
The primary objective of the present study was to develop and validate a VR-based experimental protocol enabling us to rigorously explore the effects of prolonged ( 2 hours) high-load physical and cognitive efforts. Attempting to simulate a military mission, we systematically introduced context-related cognitive tasks tapping the following competencies: memory, computation, navigation (i.e., spatial orientation), and object detection. Performance on these tasks was compared to performance on 'gold standard' cognitive assessments. Our secondary objective was to use this system to quantify cognitive and physical performance after a simulated loaded road march using a virtual environment. The present study can be viewed as an extension of previous study in which physiological and cognitive effects were studied following a 10-kilometer march (11). Using the controlled VR environment, we were able to separate out the relative contribution of cognitive load during pre-mission physical activity on physical and cognitive readiness for action immediately following the activity.

We hypothesized that VR-based cognitive tasks presented during the march would correlate with previously validated cognitive tests, and that both physical and cognitive capabilities would be affected to a greater extent after joint exposure to physical and cognitive efforts than after exposure to physical effort alone.

\section{Methods}

The study was conducted at the Heller Institute of Medical Research (The Institute for Military Physiology) and the Center of Advanced Technologies in Rehabilitation (CATR), both located at the Sheba Medical Center in Israel.

\section{Rationale}

The study was primarily designed to assess the feasibility of a VR environment-based protocol that simultaneously exposes participants to physical and cognitive demands. While the protocol includes several novel elements, the focus was on comparing new cognitive tasks presented in the context of simulated loaded military missions to conventional, validated cognitive tests (primary objective). We also conducted a pilot study to assess the effects of cognitive load during a strenuous simulated 2 hour march (pre-mission effort) on physical and cognitive performance after the exposure (secondary objective). We used a crossover trial design, with participants undergoing three activity sessions in random order, each comprising one of the following conditions: simultaneous physical and cognitive load (Phys+Cog condition), physical load only (Phys condition), and rest. For our primary objective, the outcome measure was correlation between the new VR-based cognitive tasks (during the Phys+Cog condition; see Protocol below) and a cognitive testing battery presented prior to and following the Phys+Cog condition. For the secondary objective, we conducted a within-subject pre-post comparison to determine the effects of the activity conditions on physical and cognitive performance.

\section{Participants}

Twelve healthy civilians were recruited in accordance with the following inclusion criteria: 1) male; 2) 21-30 years old; 3) served in a combat position in the military, during which they experienced loaded marches; 4) self-declared ability to endure 10-km forced walking while carrying a substantial load in a backpack; 5) above average aerobic ability (maximal oxygen uptake, $\mathrm{VO}_{2} \mathrm{max}$, above $42.4 \mathrm{ml} / \mathrm{kg} / \mathrm{min}$; (18)). Exclusion criteria included any orthopedic or other health issues. Mean $( \pm S D)$ values of the demographic variables for included participants were as follows: age $23.9 \pm 2.0$ years; weight $72.0 \pm 7.5 \mathrm{~kg}$; height $170 \pm 5 \mathrm{~cm} ; \mathrm{BMI} 24.9 \pm 1.8 \mathrm{~kg} / \mathrm{m}^{2} ; \mathrm{VO}_{2} \max 58.3 \pm 7.9 \mathrm{ml} / \mathrm{kg} / \mathrm{min}$.

Participants were informed about the study's purpose and possible risks. Their final inclusion in the study was subject to medical clearance by the study's physician and signing of an informed consent form. The study was approved by the Human Use Committee of the Sheba Medical Center (SMC-2664-15) and by the Medical Corps (IDF- 1526-15).

\section{Apparatus}

We used the Computer Assisted Rehabilitation Environment (CAREN; Motek Medical ${ }^{\odot}$, Amsterdam, the Netherlands) high-end system. The system consists of a moveable platform (3m diameter) with six degrees of freedom of movement (translations and rotations). A dual-belt instrumented treadmill is embedded within the platform. This installation is placed in a dome-shaped space. A virtual visual scene is projected on the interior surface of the dome using eight projectors, creating a $360^{\circ}$ visual display that provides a sensation of full immersion and visual depth perception. A surround sound system provides auditory stimuli congruent with the scenery. The visual flow is synchronized with the speed of the treadmill (Figure 1).

\section{Protocol}

First, each participant was invited to a preparatory visit, during which his height and weight were recorded, backpack size and weight were adjusted (see Supplementary File 1, Section 1), and a $\mathrm{VO}_{2}$ max test was performed (see Supplementary File 1, Section 2 for test description). Height (cm) was measured using a stadiometer (ADE, Germany; result $\pm 1 \mathrm{~cm}$ ), and body mass $(\mathrm{kg})$ was measured using an electronic flat scale (SECA, 803 model, Germany; result $\pm 100 \mathrm{~g}) . \mathrm{VO}_{2 \max }(\mathrm{ml} / \mathrm{kg} / \mathrm{min})$ was measured using a continuous uphill stepwise treadmill-modified Bruce protocol (19). During the $\mathrm{VO}_{2 \mathrm{max}}$ test, we recorded heart rate (HR; bpm) using the Polar RS800CX heart rate monitor (Polar ${ }^{\circledR}$, Finland). In addition, participants were familiarized with one of the baseline cognitive evaluations (SYNWIN battery, see below); they repeated the test five times with 5-minute breaks between consecutive tests. Then, as noted above, participants attended three separate activity visits, undergoing one the following protocols in each (in random order): Phys+Cog, Phys only, and rest. The physical 
component was a $10 \mathrm{~km}$ treadmill march (see details below). During the rest visit, participants sat in a room for 2 hours, during which they were exposed only to non-demanding activities such as reading a book. The time interval between visits ranged from 7 to 14 days, based on participant availability.

Each visit included the following components: a. baseline (pre-activity) assessment (see details below); b. exposure to two hours of activity/rest; and c. postactivity assessment (see details below). The pre-activity assessment included cognitive evaluation only, and the post-activity assessment included cognitive and physical evaluations. During each visit, the participants' heart rate was continuously monitored using a Polar RS800 watch with chest belt (Polar Electro, Finland), which measured the R-R time intervals.

\section{March Settings}

Participants were asked to arrive to each visit in shoes and clothing suitable for athletic activity. By combining treadmill operation profiles (speed and inclinations) with congruent visual flow speed, we simulated a $10 \mathrm{~km}$ march at a speed of $5 \mathrm{~km} / \mathrm{h}$ and $1.15^{\circ}$ slope ( $2 \%$ grade) in hilly Mediterranean terrain with nearby and distant villages (Figure 1; see also video in Supplementary File 2). To simulate diverse terrain, every 20 minutes the treadmill slope increased to $3.4^{\circ}$ ( $6 \%$ grade) for 5 minutes and then returned to the original slope. The duration of the march was exactly 2 hours. This is similar to the march settings used in previous research (11).

Participants were secured to the system in a manner that did not limit their mobility or cause discomfort (see Figure 1 and video in Supplementary File 2). They walked while carrying a backpack weighing $30 \%$ of their body weight.

In the Phys+Cog condition, participants also carried a two-way radio transceiver (walkie-talkie). Participants also had access to a drinking bag containing cold water, which was placed near the treadmill and not carried.

\section{Context-related cognitive tasks during the march}

In the Phys+Cog condition, participants performed cognitive tasks that simulated military tasks while marching, including: navigating, detecting and reporting "enemy forces" and static and dynamic objects of interest, and memorizing the status of allied forces to which they were exposed via ongoing radio transmission.

\section{Navigation}

Prior to marching, participants memorized for two minutes the navigation route based on a map simulating aerial photos of the environment (Figure $2 \mathrm{~A}$ ). Though the map was available for use during the march, they were encouraged to rely on memory as much as possible and were notified that they would be scored accordingly. The route consisted of a number of straight walking intervals of different lengths, separated by $90^{\circ}$ left and right turning points. The turning points were marked with noticeable landmarks, e.g., wells, road boards and old barrels, etc. These landmarks were also indicated on the map (Figure 2B).

Navigation was conducted by choosing a direction to turn (left or right) once a landmark was identified (total of five navigation decision points). The signal to turn was given by pointing a stick with a reflective marker attached to it, which was captured by a motion capture system (Vicon, Oxford, UK; sampling rate $120 \mathrm{~Hz}$ ). Immediately after the participant pointed to one direction, the visual scenery rotated accordingly to create the illusion of turning. If a participant chose wrong, the visual scenery rotated by 180 after 10 seconds and the participant was informed of his mistake. Feedback was consistently provided using these methods to prevent a cumulative effect from previous mistakes (see the VR-based navigation process in the video in Supplementary File 2). Scoring: each navigation decision was given a score of 1 (correct decision) or 0 (incorrect decision/did not identify the turn/looked at the map), such that total score could range from 0 to 5 .

This task primarily involved the following three cognitive functions (20)

(1) Visual and spatial memory - to memorize which land mark is associated with which turning direction.

(2) Attention and visuospatial skill - to continuously scan the changing VRE in search of memorized landmarks.

(3) Spatial orientation - to compare real landmarks to symbolic landmarks presented on the map, a cognitive task requiring spatial orientation and two dimensional to three dimensional translation.

\section{Detection and reporting of visual elements ('visual').}

Prior to the march, participants were informed that while they were walking, different objects would appear in the environment ("in the sky and on the sides of the road"), such as fighter jets, armored fighting vehicles (tanks), steel figure targets (simulating hostile combatants), and villages (see Figure 3 and video in Supplementary File 3 for visual examples). Participants were instructed to identify these objects and memorize specific information about them, such as the number and flight direction of jets (see explanation on VR compass below), the number of figure targets or armored fighting vehicles and the side on which they appeared, the number of turrets in the villages and the side of the terrain in which they appeared. To avoid misidentification, participants were shown pictures of the target objects prior to the march. Fighter jets were accompanied by a compass rose (projected on the screen in the participant's walking direction), making it easier for participants to identify their flying direction. In addition, to alert participants that jets would be passing and to improve immersion, the jets were accompanied by actual recorded jet sounds. To evaluate participant performance of these detection tasks (in a pre-defined order), the experimenter used pre-recorded questions to ask participants to report information, via the two-way radio, regarding specific, previously seen objects. When reporting detection of armored vehicles and targets, participants had to indicate the side of the road on which they appeared. No feedback was provided as to 
whether responses were correct or incorrect. Scoring: each detection question was given a score of 2 (full correct), 1 (partial correct), or 0 (incorrect), such that total score could vary between 0 and 32 .

This task primarily involved the following three cognitive functions:

(1) Visual scanning - to identify all of the aforementioned elements that were not accompanied by sound.

(2) Spatial orientation - to determine the flight direction of the fighter jets based on the virtual compass.

(3) Short term memory (21) - to store accumulated information for a few minutes and retrieve it upon request (e.g., radio call from field headquarters).

\section{Memorizing status of other allied forces ('Calc\&Mem').}

Participants were informed that there were three 30-soldier allied units walking in parallel to them, identified as the green, blue, and red units. They were also informed that they would occasionally be given updates (using the two-way radio) on the status of these forces. Each report included information about one of the units, for example, "three soldiers from the red unit have been wounded and evacuated" or, "five new soldiers have joined the blue unit." Periodically, participants were asked to provide status reports regarding the current number of soldiers in each unit, requiring them to perform calculations upon receiving the information and to memorize the updated numbers continuously throughout the march. No feedback was provided as to whether their responses were correct or incorrect. To prevent carry-over from previous mistakes, participant reports were judged (in a post-hoc evaluation) based only on the most recent responses.

Scoring: each report was given a score of 1 (correct report) or 0 (incorrect report), such that total score could vary between 0 and 9 .

This task primarily involved the following three cognitive components (22):

(1) Working memory - to continuously store information from updates on the number of soldiers in the three different allied units and periodically retrieve it to report current numbers.

(2) Short-term memory - to store the accumulated information for a few minutes and retrieve it upon request (call from field headquarters).

(3) Mathematical calculations - to add or subtract numbers to/from an existing sum.

Pre-activity and post-activity cognitive assessments

The following validated neurocognitive tasks were administered before and after the three physical activity sessions:

(1) The color version of the Trail Making Test (TMT; $(23,24,25)$, also known as the Color Trails Test (CTT), which assesses selective attention, visual and perceptual tracking abilities, and working memory $(23,24,25)$.

(2) The Synthetic Work Environment (SYNWIN) computerized test battery, which assesses short-term memory, working memory, cognitive concentration, visual perception, multitasking, reaction time, and data processing (26). The full battery comprises four sub-tasks, presented simultaneously in a 5 -minute session: a simple memory task, an arithmetic computation task, a visual monitoring task, and an auditory monitoring task. However, in the current study, we did not use the auditory monitoring task due to technical issues, as participants were not able to clearly hear tones produced by the software. The SYNWIN battery has been used in past trials to investigate the effects of various tasks and environmental factors on cognitive performance $(26,27,28,29,30)$.

To minimize learning effects while performing this battery, participants repeated the battery several times during the baseline visit. After confirming that no further score improvement was observed, the participant moved ahead with the protocol. No more than 5 repetitions were required to reach this stage.

(3) A VR version of the CTT test (17), the results of which are reported elsewhere in this issue.

Post-activity physical evaluation

We used the time to exhaustion (TTE) test only in the post-activity physical assessment, after 30 minutes of rest (during which the post-activity cognitive evaluation was conducted). The TTE [modified according to (11)] is conducted using a motor-driven treadmill, as follows: after a 2-minute warm-up (5 km/h, $2 \%$ slope), the pace and inclination was increased to match the participant's anaerobic threshold intensity (calculated from the $\mathrm{VO}_{2} \mathrm{max}$ test, see

Supplementary File 1) and maintained for 15 minutes. If the participant managed to sustain this 15 -minute stage continuously, the pace was kept constant while the inclination was elevated by $2 \%$ every 4 minutes until the participant reached subjective exhaustion. While performing the TTE test, a silicone mask was placed on the participant's face to measure respiratory values and adjusts the intensity to match his pre-defined anaerobic threshold, until he completed the first 15-min stage. The primary outcome of the TTE test was the maximum running time achieved.

\section{Outcome Measures and Data Analysis}

Outcome measures for the primary objective

We evaluated the correlations between participant scores on the validated cognitive tests and their scores on the new ecological cognitive assessment administered during the simulated march in the VR environment (VR-COG). We checked correlations with both the pre-activity and post-activity scores on the

Page 5/18 
validated cognitive tests. We also examined potential prediction of post-activity cognitive performance based on VR-COG scores.

CTT outcome measures include Part A execution time $\left(\mathrm{CTT}_{\mathrm{A}}\right)$, which measures visual and perceptual tracking and sustained attention, and Part B execution time $\left(\mathrm{CTT}_{\mathrm{B}}\right)$, which measures working memory, divided attention, sequencing skills, inhibitory control, and cognitive flexibility (23,31,32). To evaluate the effect of activity, the difference between post-activity score and pre-activity score was calculated for each part of the task $\left(\Delta \mathrm{CTT}_{\mathrm{A}}, \Delta \mathrm{CTT}_{\mathrm{B}}\right)$.

The SYNWIN produces a composite score based on performance in the three sub-tasks (a simple memory task, 'Memory', an arithmetic computation task, 'Math', and a visual monitoring task, 'Visual). To evaluate the effect of activity, the differences post-activity and pre-activity SYNWIN total and sub-task scores were calculated (SYNWIN $\Delta$ Score, $\Delta$ Memory, $\Delta$ Math, and $\Delta$ Visual monitoring).

The VR-COG outcome measures included total score for each cognitive task (navigating, detection and reporting of static and dynamic objects, and memorizing). A composite score was calculated based on all tasks using a weighted average (in accordance with the number of task-related questions that were presented to the participants).

Outcomes measures for the secondary objective

The pre-activity and post-activity cognitive and physical performance scores for all three sessions are compared and reported as preliminary data, as customary in pilot validation studies.

\title{
Statistical Analysis
}

Means \pm standard deviations (SD) are presented. To address the study primary objective of validating the new VR-COG assessments, we used Pearson correlations between the VR-COG scores and the pre-activity and post-activity cognitive assessment scores. A multiple linear regression was conducted in an attempt to predict the SYNWIN $\triangle$ Score based on the VR-COG and pre-activity CTT scores.

To address the study secondary objective, we used a Time (pre-activity and post-activity) by Condition (three visit types) repeated measures ANOVA. Post hoc pairwise comparisons were performed using Bonferroni correction $(n=3)$.

To evaluate participant fatigue in response to the different experimental conditions, a repeated measures ANOVA was used to compare time to subjective exhaustion during the TTE running test. Post hoc pairwise comparisons were performed using Bonferroni correction $(n=3)$.

Pearson correlation analysis was used to assess the correlation between HR at rest and post-rest TTE score.

Statistical significance level was set at $a=0.05$; analyses was conducted on SPSS software (SPSS Ver. 24, IBM).

\section{Results}

\section{Primary objective - Validation of the new VR-COG tests}

\author{
Correlations: VR-COG and CTT
}

Strong or medium correlations were found between three out of the four VR-COG scores (total score and sub-tasks) and at least one of the CTT component scores (Table 1). The negative correlations are in accordance with the fact that larger $\mathrm{CTT}_{\mathrm{A}}$ and $\mathrm{CTT}_{\mathrm{B}}$ are indicative of poorer performance, which higher scores on the VR-COG represent better performance.

VR-COG total score showed strong negative correlations with both the pre-activity and post-activity CTT $_{A}$ scores. VR-COG navigation score showed a strong negative correlation with the pre-activity and post-activity $\mathrm{CTT}_{\mathrm{A}}$ scores and with the pre-activity $\mathrm{CTT}_{\mathrm{B}}$ score, as well as a strong positive correlation with $\triangle \mathrm{CTT}_{\mathrm{A}}$ (indicating an activity effect). Visual VR-COG score showed a strong negative correlation with the pre-activity and post-activity CTT $_{\mathrm{A}} \mathrm{Scores} \mathrm{VR}_{\mathrm{B}} \mathrm{COG}$ Calc\&Mem scores were not correlated with the CTT components.

Table 1

Correlation coefficients (Pearson) between pre-activity and post-activity Color Trail Test (CTT) scores

\begin{tabular}{|c|c|c|c|c|c|c|}
\hline Task Type & Pre $\mathrm{CTT}_{\mathrm{A}}$ & Post $\mathrm{CTT}_{\mathrm{A}}$ & $\Delta \mathrm{CTT}_{\mathrm{A}}$ & Pre $\mathrm{CTT}_{\mathrm{B}}$ & Post $\mathrm{CTT}_{\mathrm{B}}$ & $\Delta \mathrm{CTT}_{\mathrm{B}}$ \\
\hline Total Score & $-0.75^{\star \star}$ & $-0.70^{\star}$ & 0.48 & -0.56 & -0.52 & 0.18 \\
\hline Navigation & $-0.84^{\star \star}$ & $-0.61^{*}$ & $0.69^{\star}$ & $-0.59^{\star}$ & -0.32 & 0.45 \\
\hline Visual & $-0.61^{*}$ & $-0.72^{\star *}$ & 0.28 & -0.52 & -0.57 & 0.08 \\
\hline Calc\&Mem & -0.55 & -0.54 & 0.34 & -0.39 & -0.48 & 0.00 \\
\hline
\end{tabular}


Strong or moderate positive correlations were found between all the VR-COG scores (total score and sub-tasks) and at least one of the SYNWIN component scores (Table 2). VR-COG total score showed a strong positive correlation with the pre-activity SYNWIN total score, with the post-activity SYNWIN memory subtask score, and with the pre-activity SYNWIN math sub-task score. VR-COG navigation score showed a strong positive correlation with the pre-activity SYNWIN total score and the pre-activity SYNWIN math sub-test score. VR-COG visual scores showed a moderate positive correlation with $\triangle$ Visual monitoring SYNWIN sub-task score. VR-COG Calc\&Mem score showed a strong positive correlation with the pre-activity and post-activity SYNWIN total scores, the post-activity SYNWIN memory sub-task score, and the pre-activity and post-activity SYNWIN math sub-task score. For example, Fig. 4 shows the correlation between the VR-COG Calc\&Mem score and SYNWIN math sub-task score.

Table 2

Correlation coefficients (Pearson) between pre-activity and post-activity Synthetic Work Environment (SYNWIN) battery and VR-COG scores

\begin{tabular}{|c|c|c|c|c|c|c|c|c|c|c|c|c|}
\hline Task Type & $\begin{array}{l}\text { Pre } \\
\text { SYNWIN } \\
\text { Score }\end{array}$ & $\begin{array}{l}\text { Post } \\
\text { SYNWIN } \\
\text { Score }\end{array}$ & $\begin{array}{l}\text { SYNWIN } \\
\Delta \text { Score }\end{array}$ & $\begin{array}{l}\text { Pre } \\
\text { Memory }\end{array}$ & $\begin{array}{l}\text { Post } \\
\text { Memory }\end{array}$ & $\Delta$ Memory & $\begin{array}{l}\text { Pre } \\
\text { Math }\end{array}$ & $\begin{array}{l}\text { Post } \\
\text { Math }\end{array}$ & $\Delta$ Math & $\begin{array}{l}\text { Pre } \\
\text { Visual } \\
\text { monitoring }\end{array}$ & $\begin{array}{l}\text { Post } \\
\text { Visual } \\
\text { monitoring }\end{array}$ & $\begin{array}{l}\Delta \text { Visual } \\
\text { monitoring }\end{array}$ \\
\hline $\begin{array}{l}\text { Total } \\
\text { Score }\end{array}$ & $0.77^{\star *}$ & 0.56 & 0.16 & 0.22 & $0.66^{*}$ & 0.17 & $0.78^{\star *}$ & 0.55 & 0.13 & -0.25 & 0.23 & 0.46 \\
\hline Navigation & $0.67^{\star}$ & 0.50 & 0.16 & 0.13 & 0.44 & 0.13 & $0.68^{*}$ & 0.49 & 0.14 & 0.00 & 0.28 & 0.20 \\
\hline Visual & 0.49 & 0.20 & -0.19 & 0.28 & 0.40 & -0.05 & 0.48 & 0.18 & -0.21 & -0.40 & 0.15 & $0.58^{\star}$ \\
\hline Calc\&Mem & $0.81^{\star *}$ & $0.71^{*}$ & 0.37 & 0.18 & $0.81^{* *}$ & 0.31 & $0.83^{\star *}$ & $0.69^{\star}$ & 0.33 & -0.24 & 0.18 & 0.41 \\
\hline
\end{tabular}

Predicting post-activity cognitive performance

A multiple linear regression was calculated to predict SYNWIN $\triangle$ Score based on the VR-COG tasks and the pre-activity CTT scores. A significant regression equation was found $\left(F_{(5,6)}=6.24, p=0.02, R^{2}=0.84\right)$ :

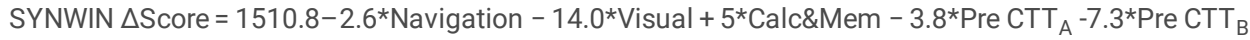

VR-COG visual $(\beta=-0.98)$, VR-COG Calc\&Mem $(\beta=0.93)$, and pre-activity $\operatorname{CTT}_{B}(\beta=-0.66)$ were significant predictors of multitasking performance, as reflected by SYNWIN $\Delta$ Score $(p=0.006, p=0.009$, and $p=0.04$, respectively). Notably, higher SYNWIN $\Delta$ Score represents better post-activity scores as compared to preactivity scores. Thus, the predictive model showed that higher navigation and visual scores during activity were associated with smaller SYNWIN improvement, and that better calculation and memory scores were associated with greater the post-activity SYNWIN scores. In addition, longer pre-activity $\mathrm{CTT}_{\mathrm{A}}$ and $\mathrm{CTT}_{\mathrm{B}}$ execution times (i.e., worse performance) were associated with lower SYNWIN $\triangle$ Score (smaller improvement). To evaluate if prediction of cognitive performance was affected by physical exhaustion, TTE score was added to the model. The addition of this component resulted in a non-significant model.

\section{Secondary objective - Activity condition effects}

\section{Cognitive evaluation}

Figure 5 presents the various CTT measures for all experimental conditions. CTT $_{\mathrm{A}}$ score showed no significant condition effect, but did show a significant time effect $\left(F_{(1,11)}=7.65, p=0.02\right)$, as the mean post-activity execution time, over conditions, was $5.2 \pm 1.9$ seconds shorter than the pre-activity execution time (better performance). Post-hoc analysis showed that $\mathrm{CTT}_{\mathrm{A}}$ score improved by $6.1 \pm 6.8$ seconds $(\mathrm{p}=0.003)$ in the rest condition but showed no significant improvement in the Phys and Phys + Cog conditions. CTT $_{B}$ score showed a significant time effect $\left(F_{(1,11)}=12.43, p=0.005\right)$, as the mean post-activity execution time, over conditions, was $4.9 \pm 1.4$ seconds shorter than the pre-activity execution time (better performance). Post-hoc analysis showed that $\mathrm{CTT}_{\mathrm{B}}$ score improved by $7.7 \pm 5.0$ seconds $(p=0.002)$ in the rest condition but showed no significant improvement in the Phys and Phys + Cog conditions. There was a significant Condition effect $\left(F_{(1.66,18.3)}=4.8, p=0.026\right)$, but post-hoc analysis showed no differences between conditions. Note that while not significant $(p=0.08)$, it seems that the added cognitive effort in the Phys + Cog condition tended to lessen the improvement in $\mathrm{CTT}_{B}$ associated with physical effort alone (51.03 \pm 3.4 seconds). Similarly, evaluation of mean change over time for the three conditions in both CTT execution times (i.e., $\Delta \mathrm{CTT}_{\mathrm{A}}$ and $\Delta \mathrm{CTT}_{\mathrm{B}}$ ) revealed no significant differences.

Figure 6 presents the SYNWIN composite scores for all three conditions, before and after activity. Only a significant Time effect was found $(F(1,11)=6.64, p=$ 0.026). In all conditions, composite score tended to be higher post-activity than it was pre-activity (rest: $833 \pm 255$ vs. $878 \pm 244$ pts., Phys: $828 \pm 200$ vs. $885 \pm$ 189 pts., and Phys + Cog: $870 \pm 177$ vs. $889 \pm 286$ pts., respectively). However, post-hoc analysis showed no significant time differences in each condition separately.

Physical evaluation

As expected, both physical conditions elicited higher HR values than the rest condition (rest: $57 \pm 2$ bpm, Phys: $107 \pm 8$ bpm, Phys + Cog: $108 \pm 8$ bpm, $p<$ 0.0005). No significant difference was found between HR values during both physical efforts. To evaluate fatigue responses to the different experimental 
conditions, times to subjective exhaustion during the TTE running test were compared. Due to a technical problem in one of the experiments, the TTE analysis was based only on 11 participants. A significant Condition effect was found $(F(2,20)=3.76, p=0.04)$. As expected, post-rest TTE tended to be higher than post-activity TTE for the two exercise conditions (rest: $22.06 \pm 3.34$ minutes, Phys: $20.36 \pm 5.13$ minutes, Phys + Cog: $20.56 \pm 4.95$ minutes). However, post hoc analysis showed no significant differences between each two conditions. These results indicate that while both physical efforts caused a greater physiological response, as indicated by the HR values, cognitive effort did not cause greater post-exercise fatigue. Pearson correlation analysis showed a strong-negative correlation between HR during the two hours of physical activity and post-activity TTE score in the Phys and the Phys + Cog conditions $(r=$ $-0.72, p=0.019 ; r=-0.74, p=0.015$, respectively), but not in the rest condition. These findings indicate that participants who experienced higher heart rates (i.e., greater intensity) while walking, tended to reach physical fatigue earlier (reduced TTE).

\section{Discussion}

We describe a VR environment that was developed to validate a novel experimental protocol involving both physical and cognitive loads, the latter of which was achieved using context-related cognitive tasks that could then be compared with 'gold standard' cognitive tasks. Using this protocol in an initial pilot study, we assessed the effects of strenuous physical and cognitive activity on post-activity physical and cognitive performance. Our VR environment and props simulated a military march incorporating cognitive tasks designed to mimic actual military tasks.

VR enables participants to experience situations under ecologically-valid but controlled conditions $(16,33)$. Special sceneries can be created for different professionals reflecting specific ecological elements associated with each profession's requirements. Thus, behavioral responses studied under VR settings are closely 'faithful' to the anticipated behavior in the daily natural settings, supporting the external validity of the VR based observations.

Tasks introduced during our protocol correlated with existing, validated cognitive tests, providing a more comprehensive and ecologically valid tool for assessing physical and cognitive abilities. We believe that it can be customized to assess rescue personnel and athletes who are required to perform combined intense physical and cognitive activities for extended periods of time.

\section{Summary of main findings}

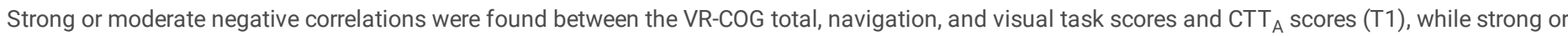
moderate positive correlations were found between all the VR-COG scores and at least one of the SYNWIN component scores. These findings support the feasibility of alternative context-related VR-based testing schemes that measure cognitive constructs common to traditional, validated tests. Together, the VRCOG and CTT scores successfully predicted the effects of combined physical and cognitive load on multitasking performance, as reflected by the change in SYNWIN performance.

The secondary objective of the study was to compare the two activity conditions, to determine the added effect of cognitive activity. Physical activity was found to elicit some physiological exhaustion compared to the rest condition, as expressed by the TTE test results. However, adding a cognitive load did not cause an additional change in TTE score.

Regards the comparison between pre-activity and post-activity cognitive assessments, it appeared that learning effects overshadowed any potential effect of activity condition (i.e., physical and cognitive versus physical alone), as the SYNWIN and CTT outcome measures showed no condition effects but did show significant time effects, indicating better performance in the post-activity than the pre-activity assessments. The one exception was the condition effect found for $\mathrm{CTT}_{\mathrm{B}}$, suggesting better performance after activity than after rest.

\section{Validation of the ecological VR environment}

Our VR application was designed to assess performance on various cognitive demands expected from high performance professionals such as soldiers, rescue personnel, and athletes, including sustained attention, visual perception, and integration of information from multiple sources. The application is unique in allowing evaluation of these cognitive abilities during exercise.

We found a strong correlation between VR-COG total score and CTT $\mathrm{A}$ (Table 1). Shorter CTT $_{\mathrm{A}}$ execution time indicates better visuo-perceptual performance. The fact that the navigation and visual sub-tasks, but not the Calc\&Mem sub-task, contributed to this correlation supports our assertion that these sub-tasks primarily evaluate visuo-perceptual abilities $(23,31)$.

Interestingly, the navigation sub-task was also correlated with pre-activity $\mathrm{CTT}_{\mathrm{B}}$, most likely because both require decision-making based on visual input. The $\mathrm{CTT}_{\mathrm{B}}$ test involves, for example, divided attention, cognitive alternation, and inhibition control. These abilities are also tapped during the navigation task, in which participants must scan their environment and make decisions based on a particular landmark. In contrast, the Calc\&Mem sub-task involves cognitive functions, such as mathematical calculations, that are not involved in CTT performance.

These findings were corroborated by the correlations between the VR-COG measures and the SYNWIN test (Table 2). While the VR-COG total score and navigation and Calc\&Mem sub-tasks were correlated with SYNWIN total score and other sub-tasks, VR-COG visual score was correlated only to the visual monitoring task, which relies on a specific, similar cognitive ability. It is noteworthy that the SYNWIN battery was designed to evaluate multitasking, as different sub-tasks are presented simultaneously. It appears that this effect was achieved more ecologically and naturally using our new VR application. Immersion in the VR environment, continually changing visual content, and tasks presented over a relatively longer time period inherently enable the VR-COG to assess multitasking ability. 
While exerting themselves physically in the VR environment, participants were required to process and memorize information from multiple sources until they were required to report about it. In the meanwhile, they performed other cognitive tasks (i.e., navigation and calculation). Thus, working memory was required in all the VR-COG tasks. Indeed, strong relationships between working memory and multitasking have been documented in the past (34, 35), and specifically working memory was found related to the performance on the SYNWIN battery (34).

\section{Predicting post-activity cognitive performance}

To evaluate the combined effect of physical and cognitive load on cognitive performance, we chose the SYNWIN $\triangle$ Score as our examination parameter (see Eq. 1). Our findings confirm the feasibility of quantifying predicted effects on cognitive performance based on the VR-COG battery and $\mathrm{CTT}_{\mathrm{B}}$ (which have similarities).

\section{The effect of strenuous marching and simultaneous cognitive load on cognitive performance}

Cognitive performance as assessed by the CTT and SYNWIN tests was not affected by either exercise protocol, while CTT scores alone improved following the rest condition, possibly due to a learning effect (Fig. 5). While improvement was recorded in the post-activity SYNWIN assessment, as compared to the preactivity assessment, the effect was not statistically significant (Fig. 6). Post hoc analysis showed that the visits order did not have any effect on the participants' results. Based on this and on the fact that we took the measure of acclimatizing the participant to the SYNWIN battery (i.e., several trials prior to the start of the protocol; see Methods), we rule out that the post-pre comparison was affected by learning.

In attempting to explain the lack of condition effects, we address aerobic fitness, timing of cognitive evaluation, and exercise duration. Participants recruited for this study presented high aerobic fitness (high $\mathrm{VO}_{2} \mathrm{max}$ ), which made the physical load exercise intensity to be light to moderate for them ( $\sim 50 \%$ of $\mathrm{VO}_{2} \mathrm{max}$ ). It has previously been shown that exercise of light to moderate intensity has no appreciable effects on cognition. Moreover, athletes are generally not expected to present with cognitive changes during exercise of any intensity, while less fit participants have shown better cognitive performance after moderate, but not light, exercise $(4,36,37)$.

Regarding the timing of evaluation, it has been suggested that a 0-10-minute break following a single bout of exercise will not cause improvement in cognitive functions, while a break longer than 11 minutes might lead to significant improvement $(38,39)$. In our experiment, cognitive assessment was performed directly after the physical activity.

Another prominent factor potentially affecting the relationship between exercise and cognitive performance is exercise duration. It has been propose that exercise durations greater than one hour are needed to significant effect cognitive performance, due to fatigue $(5,40)$. While our protocol lasted two hours, given our participants' fitness level, it was apparently not enough to evoke significant fatigue.

It should be noted, however, that for some participants, the combined cognitive and physical load tended to lower cognitive performance, while for others it appeared to cause some degree of improvement. Further research is needed to address potential variance in response to this kind of activity (i.e., combined cognitive and physical load).

\section{Limitations and future directions}

We did not assess the extent to which VR simulation create high cognitive load during the march. This can be accomplished by evaluating effects on gait and on autonomic nervous system responses. In real-world military scenarios, soldiers experience cognitive load while in stressful environments. Therefore, the interaction between exercise and cognitive performance among armed forces should also be evaluated in extreme scenarios with a greater number of stressors, to more closely represent real field conditions. In this regard, both VR applications and potential real world scenarios should be adapted to different high-performance professional contexts. For example, a VR-COG battery designed for firefighters should contain elements found in their professional environment.

Finally, while suitable for our goal of a pilot feasibility and validation study, the sample size was too small to draw solid conclusions about the effects on cognitive performance of physical exercise alone and in combination with cognitive load. In particular, to delineate different type of 'responders' to the exposures, i.e., those who show improvements vs. those who show decline in the post activity evaluations. It is also noted that the VR-COG approach can potentially assess changes in cognitive performance during physical stress.

\section{Conclusions}

Large scale VR-based applications provide highly immersive environments and controlled platforms for simulating scenarios experienced by highperformance professionals. The present work focused on military-oriented tasks.

The study established a setup for assessing the combined effects of physical and cognitive effort on later physical and cognitive performance, thereby contributing to existing knowledge regarding motor-cognitive interactions. In particular, the VR-COG approach can potentially assess changes in cognitive performance under physically stressful conditions, as the tests are presented during activity. The proposed application can be used to screen highperformance professionals. 


\section{Abbreviations}

\section{CAREN}

Computer Assisted Rehabilitation Environment

CATR

Center of Advanced Technologies in Rehabilitation

CTT

Color Trail Test; HR - heart rate

Phys

activity condition with physical load only

Phys + Cog

activity condition with simultaneous physical and cognitive load

SYNWIN

The Synthetic Work Environment computerized test battery

SD

standard deviation

TMT

Trail Making Test

TTE

Time to Exhaustion test

VR

Virtual reality

VR-COG

cognitive tasks in virtual reality settings

VRE

VR environment

\section{Declarations}

\section{Acknowledgements}

We thank Mr. Hani Baransi from the Center of Advanced Technologies in Rehabilitation for technical support, and Ms. Michelle Florentine and Mr. Ryan Eli Solymani for English editing.

\section{Ethics approval and consent to participate}

All participants in the study provided their written informed consent to participate. The research protocol was approved by the Sheba Medical Center Human Studies Committee (SMC-2664-15).

\section{Consent for publication}

Not applicable

\section{Availability of data and materials}

The data supporting the findings of this study are available upon request from the corresponding author. Data are not publicly available due to privacy or ethical restrictions.

\section{Competing interests}

The authors declare that they have no competing interests.

\section{Funding}

This study was supported in part by the Israeli Ministry of Defense Grant \#4440939339

\section{Authors' contributions}

Recruitment of the participants - IK; Running the experiments - IK, SKN; Analyzing the data - IK, SKN, MP; Drafting the manuscript - IK, SKN, AG, MP; Study design - RY, YH, MP; Building and designing the VR environment - YB; Operating the experiments- YB, OBG; Video editing - OBG.

\section{References}

1. Brisswalter J, Collardeau M, René A. Effects of acute physical exercise characteristics on cognitive performance. Sports medicine. 2002;32(9):555-66.

2. Harrison Y, Horne JA. The impact of sleep deprivation on decision making: a review. Journal of experimental psychology: Applied. 2000;6(3):236. 
3. Kavanagh J. Stress and Performance A Review of the Literature and its Applicability to the Military. RAND CORP SANTA MONICA CA, 2005.

4. Labelle V, Bosquet L, Mekary S, Bherer L. Decline in executive control during acute bouts of exercise as a function of exercise intensity and fitness level. Brain cognition. 2013;81(1):10-7.

5. Dietrich A, Audiffren M. The reticular-activating hypofrontality (RAH) model of acute exercise. Neuroscience Biobehavioral Reviews. 2011;35(6):1305-25.

6. Dietrich A. The transient hypofrontality theory and its implications for emotion and cognition. Exercise and cognitive function2009.

7. Nindl BC, Barnes BR, Alemany JA, Frykman PN, Shippee RL, Friedl KE. Physiological consequences of US Army Ranger training. Med Sci Sports Exerc. 2007;39(8):1380-7.

8. Sams ML. Comparison of static and countermovement jump variables in relation to estimated training load and subjective measures of fatigue. 2014.

9. Ziemann E, Grzywacz T, Luszczyk M, Laskowski R, Olek RA, Gibson AL. Aerobic and anaerobic changes with high-intensity interval training in active college-aged men. The Journal of Strength Conditioning Research. 2011;25(4):1104-12.

10. Sobhani V, Hajizadeh B, Bazgir B, Kazemipour M, Shamsodini A, Shakibaey A. Effect of 8-week omega-3 supplementation on pulmonary function during classic army ranger training. Feyz Journal of Kashan University of Medical Sciences. 2014;17(6).

11. Yanovich R, Hadid A, Erlich T, Moran DS, Heled Y. Physiological and cognitive military related performances after 10-kilometer march. Disaster Military Medicine. 2015;1(1):6.

12. Eddy MD, Hasselquist L, Giles G, Hayes JF, Howe J, Rourke J, et al. The effects of load carriage and physical fatigue on cognitive performance. PloS one. 2015;10(7):e0130817.

13. Armstrong N, Doyle D, Smith S, Risius D, Wardle S, Greeves JP et al, editors. A preliminary study of the effects of load carriage on cognition during a simulated military task in male and female soldiers. 4th International Congress on Soldiers' Physical Performance; 2017.

14. Parsons TD. Virtual reality for enhanced ecological validity and experimental control in the clinical, affective and social neurosciences. Front Hum Neurosci. 2015;9:660.

15. Cano Porras D, Sharon H, Inzelberg R, Ziv-Ner Y, Zeilig G, Plotnik M. Advanced virtual reality-based rehabilitation of balance and gait in clinical practice. Therapeutic advances in chronic disease. 2019;10:2040622319868379.

16. Porras DC, Siemonsma P, Inzelberg R, Zeilig G, Plotnik M. Advantages of virtual reality in the rehabilitation of balance and gait: systematic review. Neurology. 2018;90(22):1017-25.

17. Plotnik M, Doniger GM, Bahat Y, Gottleib A, Gal OB, Arad E et al, editors. Immersive trail making: Construct validity of an ecological neuropsychological test. Virtual Rehabilitation (ICVR), 2017 International Conference on; 2017: IEEE.

18. Astrand I. Aerobic capacity in men and women with special reference to age. Acta physiol scand. 1960;49(169):1-89.

19. Bruce R. Multi-stage treadmill test of submaximal and maximal exercise. Exercise testing and training of apparently healthy individuals: a handbook for physicians. 1972:32-4.

20. Moffat SD. Aging and spatial navigation: what do we know and where do we go? Neuropsychology review. 2009;19(4):478.

21. Xu Y, Chun MM. Dissociable neural mechanisms supporting visual short-term memory for objects. Nature. 2006;440(7080):91-5.

22. Tombaugh TN. A comprehensive review of the paced auditory serial addition test (PASAT). Archives of clinical neuropsychology. 2006;21(1):53-76.

23. D'Elia L, Satz P, Uchiyama C, White T. Color trails test, psychological assessment resources. Florida, USA: Psychological Assessment Resources (PAR) Wechsler Memory Scale. 1989.

24. Reitan RM. Validity of the Trail Making Test as an indicator of organic brain damage. Percept Mot Skills. 1958;8(3):271-6.

25. Reitan RM, Wolfson D. Category Test and Trail Making Test as measures of frontal lobe functions. The Clinical Neuropsychologist. 1995;9(1):50-6.

26. Elsmore TF. SYNWORK1: A PC-based tool for assessment of performance in a simulated work environment. Behav Res Methods. 1994;26(4):421-6.

27. Braude D, Goldsmith T, Weiss SJ. Assessing air medical crew real-time readiness to perform critical tasks. Prehospital Emergency Care. 2011;15(2):25460.

28. Matsangas P, McCauley ME, Becker W. The effect of mild motion sickness and sopite syndrome on multitasking cognitive performance. Human factors. 2014;56(6):1124-35.

29. Barron LG, Rose MR. Multitasking as a Predictor of Pilot Performance: Validity Beyond Serial Single-Task Assessments. Military Psychology. 2017;29(4):316-26.

30. Beer J, Shender BS, Chauvin D, Dart TS, Fischer J. Cognitive deterioration in moderate and severe hypobaric hypoxia conditions. Aerospace medicine human performance. 2017;88(7):617-26.

31. Sanchez-Cubillo I, Perianez J, Adrover-Roig D, Rodriguez-Sanchez J, Rios-Lago M, Tirapu J, et al. Construct validity of the Trail Making Test: role of taskswitching, working memory, inhibition/interference control, and visuomotor abilities. J Int Neuropsychol Soc. 2009;15(3):438-50.

32. Arbuthnott K, Frank J. Trail making test, part B as a measure of executive control: validation using a set-switching paradigm. J Clin Exp Neuropsychol. 2000;22(4):518-28.

33. Rizzo A, Parsons TD, Lange B, Kenny P, Buckwalter JG, Rothbaum B, et al. Virtual reality goes to war: A brief review of the future of military behavioral healthcare. J Clin Psychol Med Settings. 2011;18(2):176-87.

34. Hambrick DZ, Oswald FL, Darowski ES, Rench TA, Brou R. Predictors of multitasking performance in a synthetic work paradigm. Applied cognitive psychology. 2010;24(8):1149-67.

35. Redick TS, Shipstead Z, Meier ME, Montroy JJ, Hicks KL, Unsworth N, et al. Cognitive predictors of a common multitasking ability: Contributions from working memory, attention control, and fluid intelligence. J Exp Psychol Gen. 2016;145(11):1473.

Page 11/18 
36. Hüttermann S, Memmert D. Does the inverted-U function disappear in expert athletes? An analysis of the attentional behavior under physical exercise of athletes and non-athletes. Physiol Behav. 2014;131:87-92.

37. Pontifex MB, Hillman CH, Fernhall B, Thompson KM, Valentini TA. The effect of acute aerobic and resistance exercise on working memory. Med Sci Sports Exerc. 2009;41(4):927-34.

38. Chang Y-K, Labban J, Gapin J, Etnier JL. The effects of acute exercise on cognitive performance: a meta-analysis. Brain research. 2012;1453:87-101. 39. Soga K, Shishido T, Nagatomi R. Executive function during and after acute moderate aerobic exercise in adolescents. Psychol Sport Exerc. 2015;16:7-17.

40. Davranche K, Brisswalter J, Radel R. Where are the limits of the effects of exercise intensity on cognitive control? Journal of Sport Health Science. 2015;4(1):56-63.

\section{Supplemental File Legends}

\section{Supplementary File 1}

\section{Section 1 - Load carrying conditions}

Military backpack (MB): A standard 90L military backpack (Lowe Alpine, UK) was used. To achieve maximal homogeneous load distribution, we used plastic water packs. Most of the weight was placed in the central compartment of the backpack (located close to the participant's back), as packing heavy items high in the backpack could be the most energy-efficient method of carrying a load on the back (1). After loading the backpack with a weight equal to $30 \%$ of the participant's bodyweight, it was adjusted according to the participant's height and the straps (waist and shoulder) were adjusted to maximize subjective comfort. A load of $30 \%$ of body weight was used because it is the normative load carried by most soldiers according to current Israel Defense Force (IDF) guidelines.

\section{Section $2-\mathrm{VO}_{2} \max$}

We followed a conventional procedure for evaluating aerobic ability. Maximal oxygen uptake $\left(\mathrm{VO}_{2} \mathrm{max}, \mathrm{ml} / \mathrm{kg} / \mathrm{min}\right)$ was measured using a continuous uphill stepwise treadmill modified Bruce protocol (2), using a metabolic system (CPET ZAN600, nSPIRE). Oxygen uptake was measured using a breath-by-breath mask with a ZAN 600 ergo test system (ZAN Messgeräte $\mathrm{GmbH}$, Oberthulba, Germany). The protocol consists of a 5 -minute resting period followed by 5 minutes of warm-up walking at $5 \mathrm{~km} / \mathrm{h}$ and $2 \%$ grade (using a treadmill). Then, speed is increased to $9 \mathrm{~km} / \mathrm{h}$, and then by $1 \mathrm{~km} / \mathrm{h}$ every $2 \mathrm{minutes}$ until volitional fatigue. After reaching maximal capacity, the participant continues walking for 3 minutes for active recovery and then rests until reaching full physiological recovery.

\section{References:}

1. Stuempfle KJ, Drury DG, Wilson AL. Effect of load position on physiological and perceptual responses during load carriage with an internal frame backpack. Journal of Ergonomics. 2004; 45(7):784-789.

2. Bruce R. Multi-stage treadmill test of submaximal and maximal exercise. Exercise testing and training of apparently healthy individuals: a handbook for physicians. 1972:32-4.

\section{Supplementary File 2}

The video shows an example of one participant in protocol setup preforming the physical activity condition. Ten Km march in simulated hilly Mediterranean terrain with nearby and distant villages with changing slopes. Participants were secured to the system in a manner that did not limit their mobility or cause discomfort. They walked while carrying a backpack weighing $30 \%$ of their body weight.

\section{Supplementary File 3}

The video demonstrates all VR based cognitive tasks that were introduced to the participants during the Physical and Cognitive activity conditions.

\section{Figures}




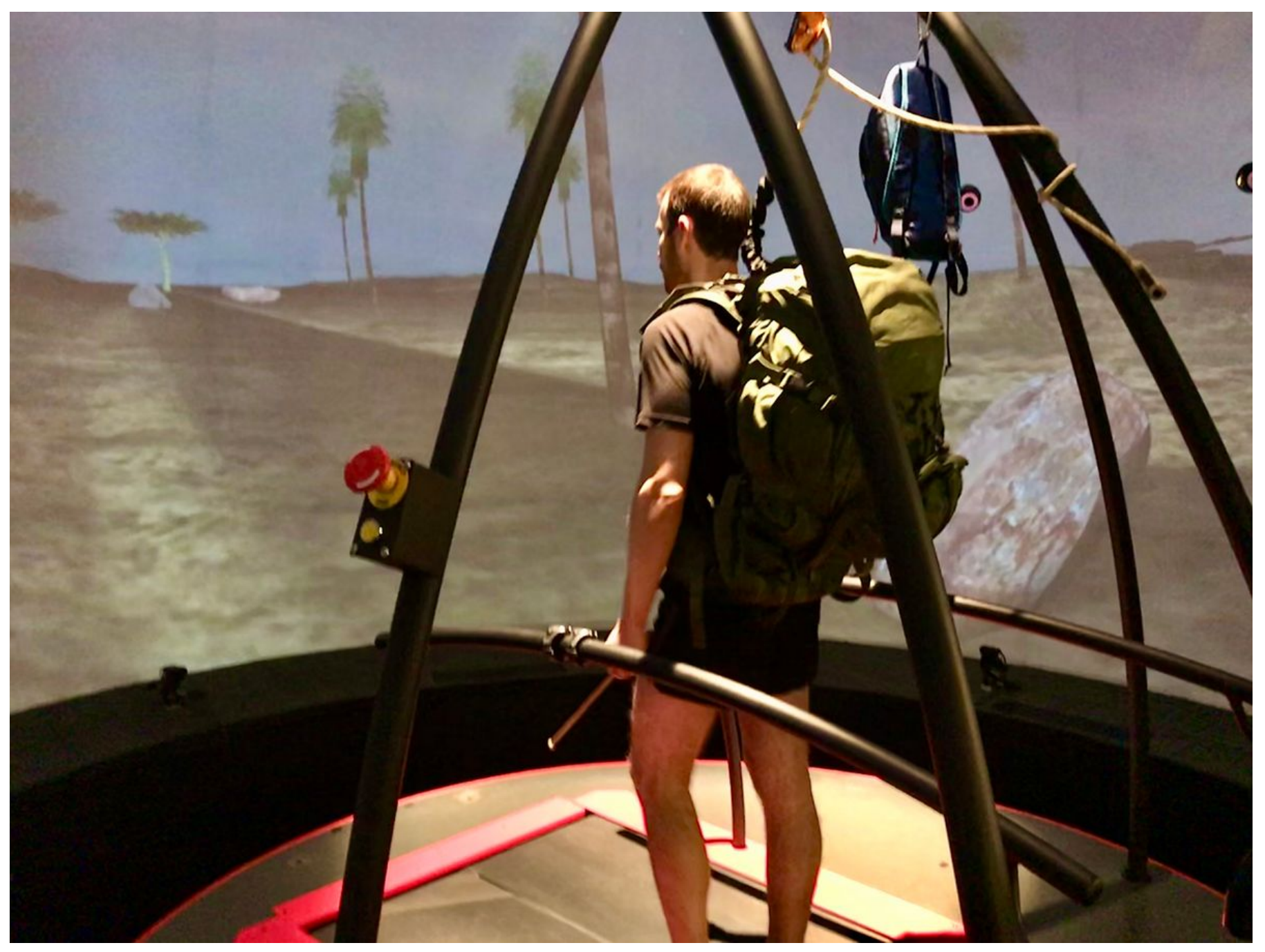

\section{Figure 1}

The Computer Assisted Rehabilitation Environment (CAREN) is a fully immersive virtual reality system with a 6 degrees of freedom moveable platform, synchronized with virtual visual scenery projected over a $360^{\circ}$ dome-shaped screen. A treadmill is embedded in the platform and its speed is synchronized with the visual flow. A surround sound system provides auditory stimuli congruent with the scenery. A motion capture system (Vicon) and set of force plates in the treadmill provide performance data. In the current study, participants walked while carrying a backpack weighing $30 \%$ of their body weight, and were safely secured to the system during the entire march. A bag of drinking water was available (hung on the upper right side of the metal frame). 


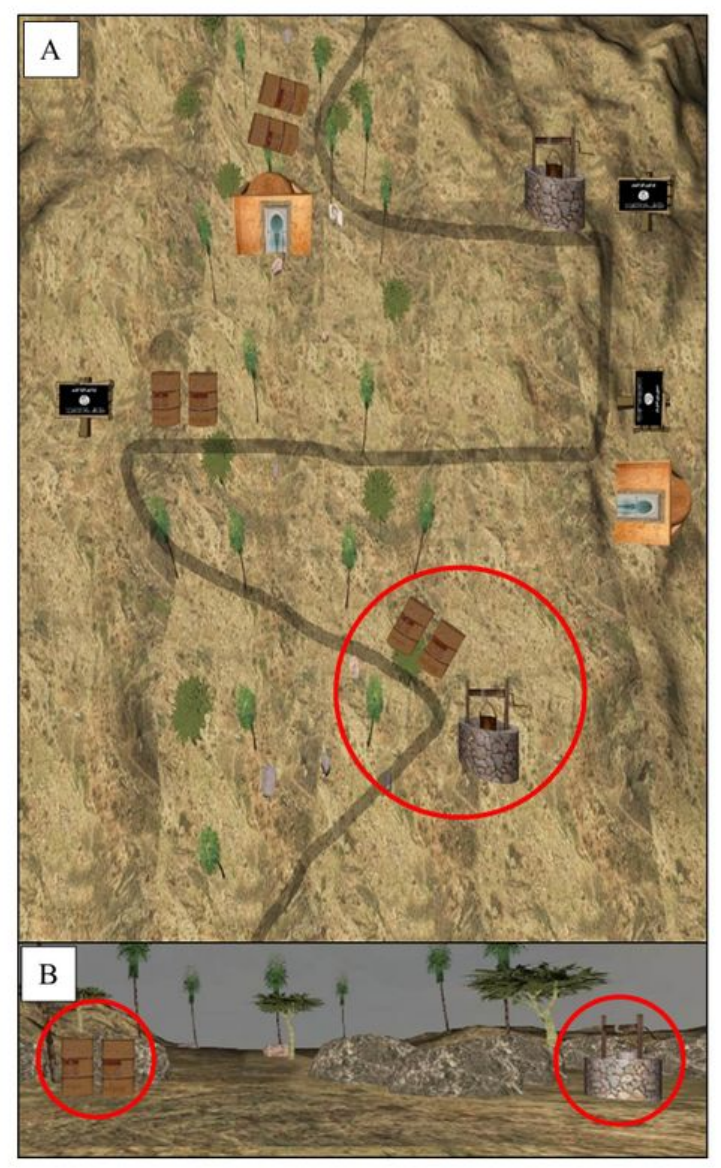

\section{Figure 2}

A. Schematic map of the march route. B. Example of a well and barrels (marked with red circles) marking the first turn. 


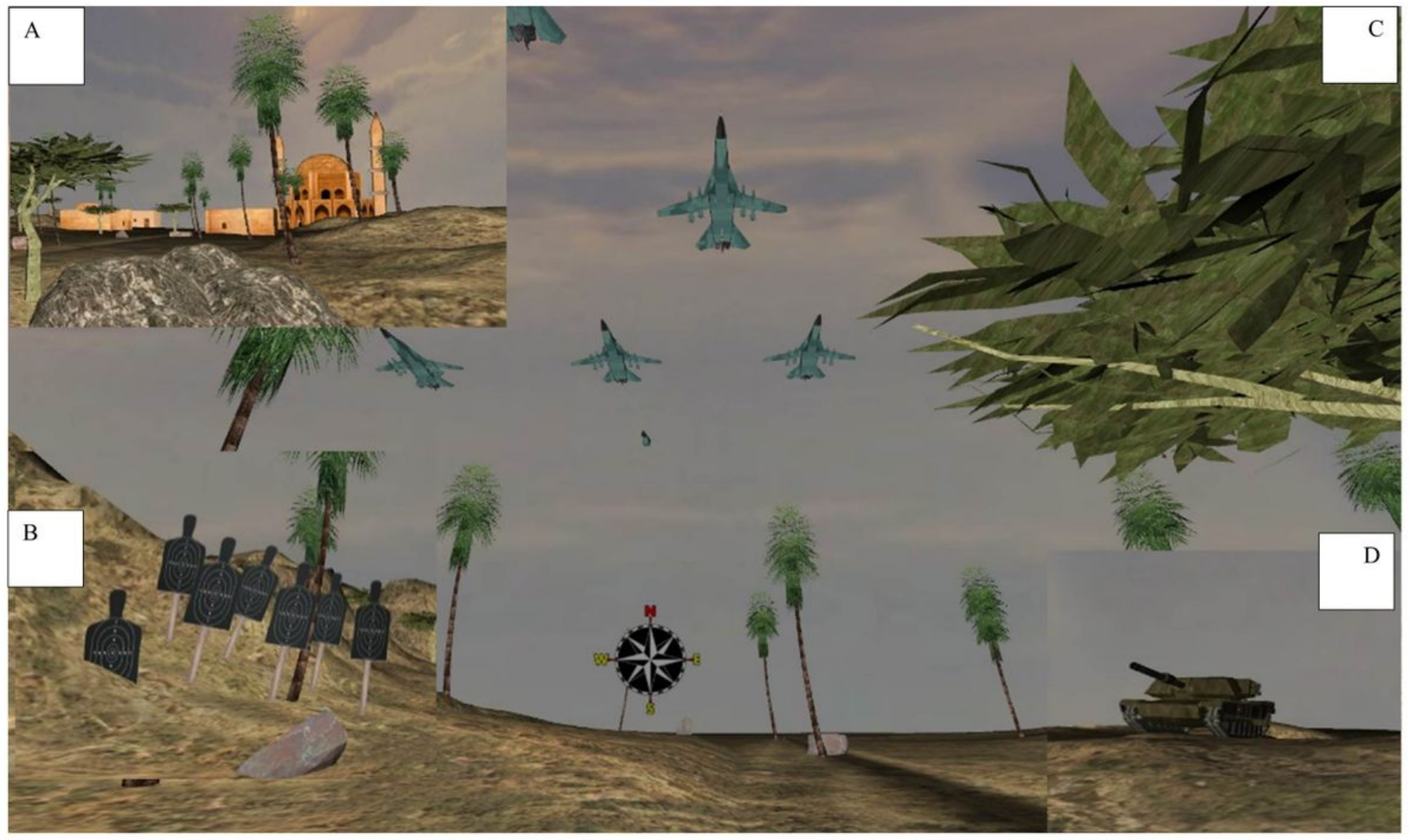

\section{Figure 3}

Examples of the visual elements for detection during the march. A. A typical Mediterranean village. B. Steel figure targets. C. Fighter jets with a compass rose. D. armored fighting vehicle ('tank'). 


\section{Correlation between SYNWIN Math Sub-Task and VR-COG Calc\&Mem Scores}

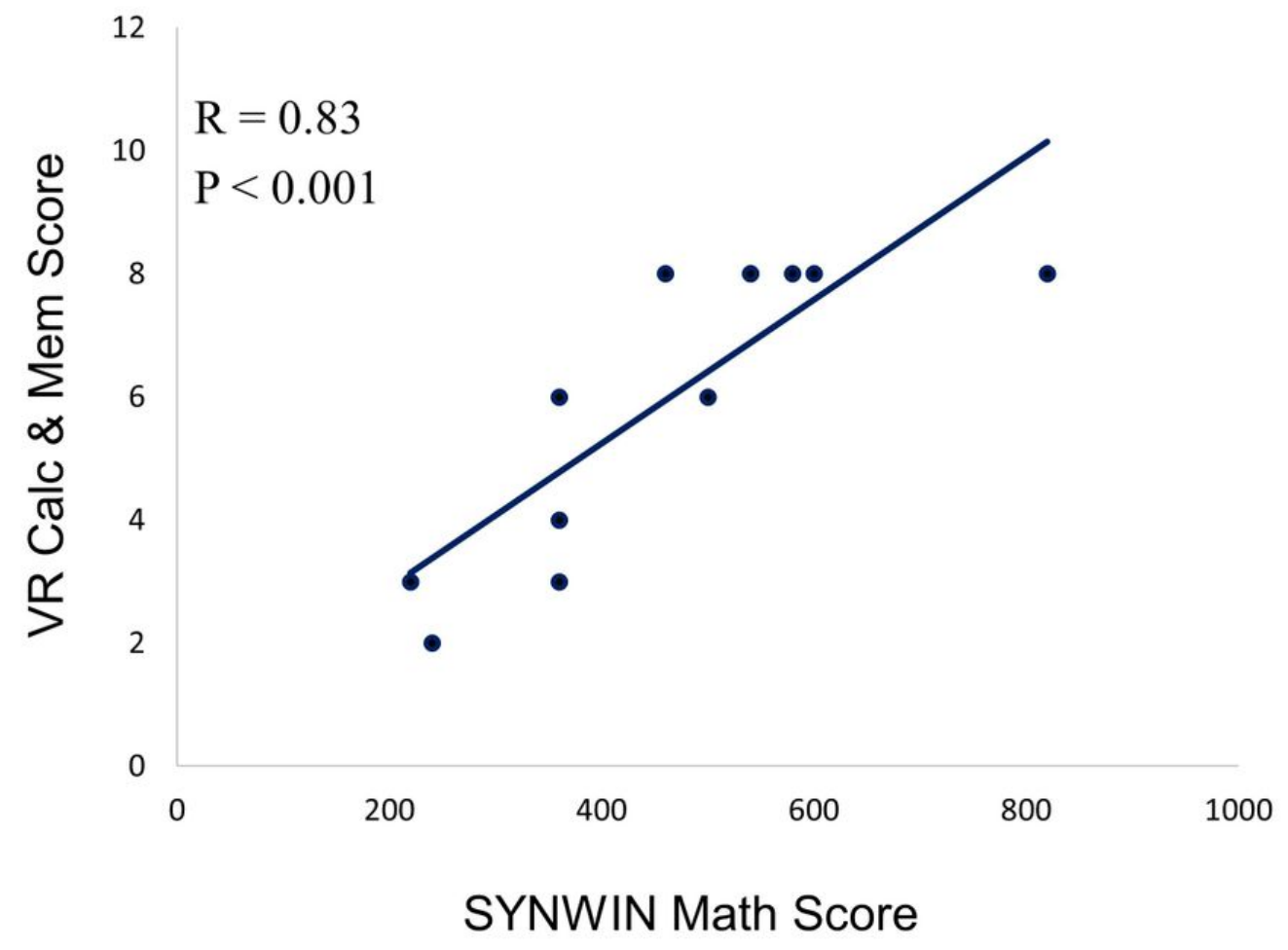

Figure 4

Correlation analysis (Pearson) between the Synthetic Work Environment (SYNWIN) math score and the VR-COG Calc\&Mem score. Due to the relatively discrete nature of the values, we also conducted Spearman correlation analysis and found significant correlations $($ Spearman roh $=0.87, p<0.001)$. 

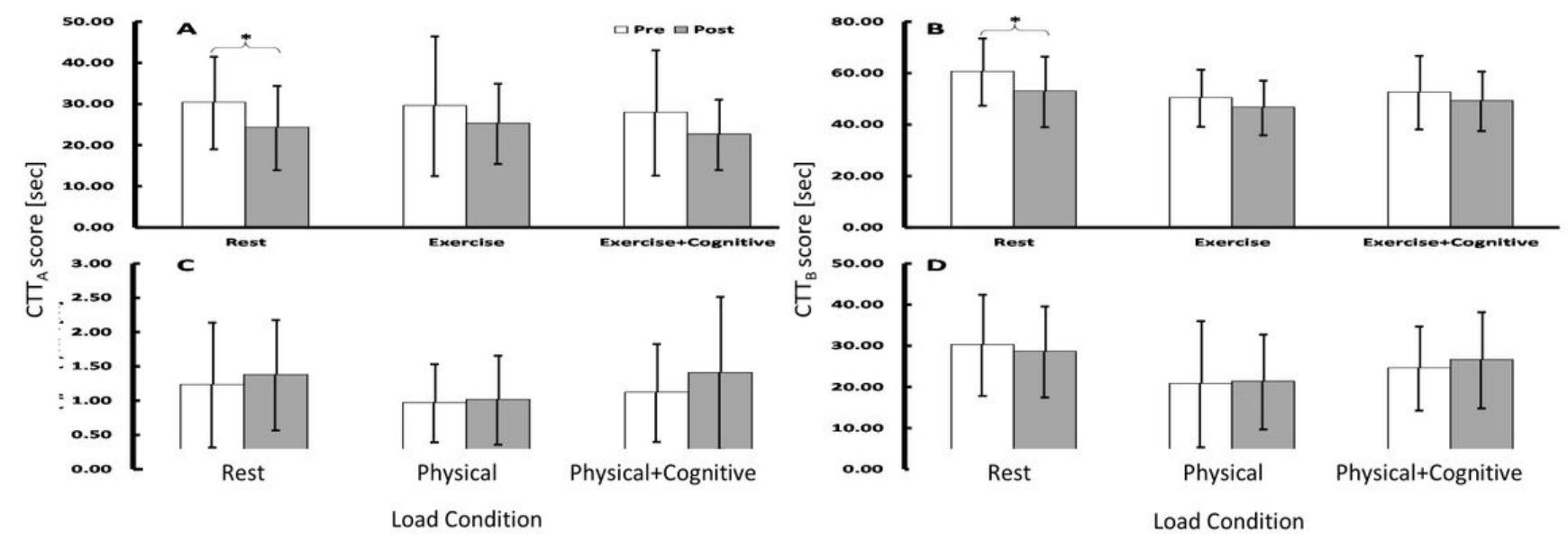

Figure 5

Pre-activity and post-activity CTTA (A) and CTTB (B) scores for all experimental conditions; ${ }^{\mathrm{p}}<<0.05$. 


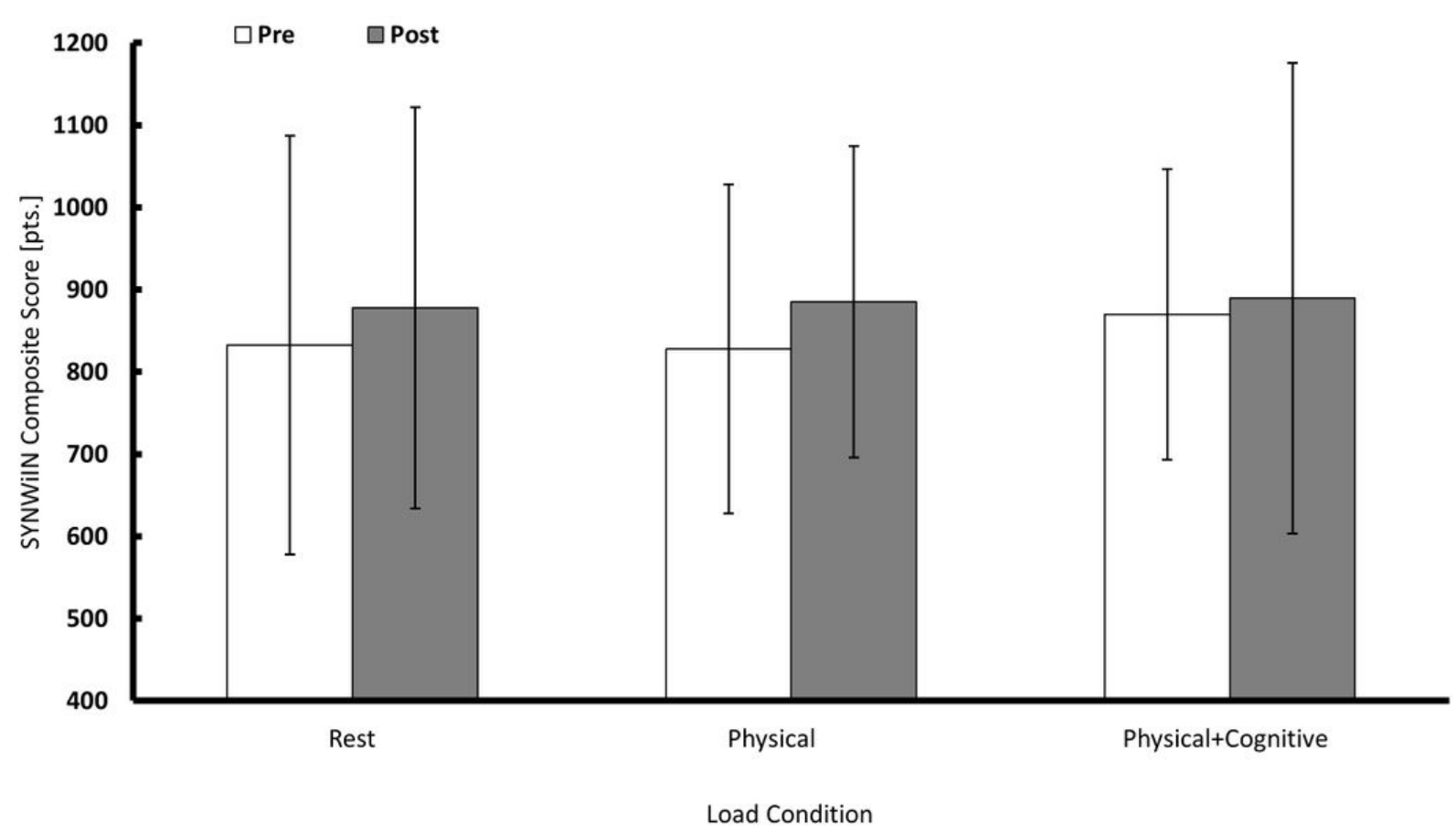

Figure 6

Pre-activity and post-activity SYNWIN composite scores for all experimental conditions.

\section{Supplementary Files}

This is a list of supplementary files associated with this preprint. Click to download.

- SupplementaryFile3.mp4

- SupplementaryFile2.mp4

- SupplementaryFile1.docx 